Annals of Pure and Applied Mathematics

Vol. 19, No.1, 2019, 1-5

ISSN: 2279-087X (P), 2279-0888(online)

Published on 10 January 2019

www.researchmathsci.org

DOI: http://dx.doi.org/10.22457/apam.594v19n1al

Annals of

Pure and Applied

Mathematics

\title{
On Solutions of the Diophantine Equations $p^{4}+q^{4}=z^{2}$ and $p^{4}-q^{4}=z^{2}$ when $p$ and $q$ are Primes

\author{
Nechemia Burshtein
}

117 Arlozorov Street, Tel - Aviv 6209814, Israel

Email: anb17@netvision.net.il

Received 2 January 2019; accepted 9 January 2019

Abstract. In this article, we consider the equations $p^{4}+q^{4}=z^{2}$ and $p^{4}-q^{4}=z^{2}$ when $p, q$ are primes and $z$ is a positive integer. We establish that $p^{4}+q^{4}=z^{2}$ has no solutions for all primes $2 \leq p<q$. For $p^{4}-q^{4}=z^{2}$ when $2 \leq q<p$, it is shown: (i) For $q=2$ the equation has no solutions. (ii) The equation $z^{2}=p^{4}-q^{4}=\left(p^{2}-q^{2}\right)\left(p^{2}+q^{2}\right)$ is impossible when each factor is equal to a square. (iii) When each factor is not a square, conditions which must be satisfied simultaneously are determined for $p^{2}$ and $q^{2}$. For all primes $p<2100$, these conditions are not fulfilled simultaneously. It is conjectured for all primes $p>2100$ and $q>2$ that the equation has no solutions.

Keywords: Diophantine equations

\section{AMS Mathematics Subject Classification (2010): 11D61}

\section{Introduction}

The field of Diophantine equations is ancient, vast, and no general method exists to decide whether a given Diophantine equation has any solutions, or how many solutions. In most cases, we are reduced to study individual equations, rather than classes of equations.

The famous general equation

$$
p^{x}+q^{y}=z^{2}
$$

has many forms. The literature contains a very large number of articles on non-linear such individual equations involving primes and powers of all kinds. Among them are [2, $3,4]$ and others.

In this paper, we consider the two equations

$$
\begin{gathered}
p^{4}+q^{4}=z^{2}, \\
p^{4}-q^{4}=z^{2}
\end{gathered}
$$

where $p, q$ are primes, and $z$ is a positive integer. All other values denote positive integers too. 


\section{Nechemia Burshtein}

In the two self-contained Sections 2 and 3 , the two equations are respectively investigated for solutions. In Section 2, we show for all primes $2 \leq p<q$ that $p^{4}+q^{4}=$ $z^{2}$ has no solutions. In Section 3, we consider $p^{4}-q^{4}=z^{2}$, and prove that when $q=2$ and when $2<q<p<2100$ the equation has no solutions. In our proof we use "Pythagorean triples".

\section{Solutions of the equation $p^{4}+q^{4}=z^{2}$}

For all primes $p, q$, it is shown in the following theorem that $p^{4}+q^{4}=z^{2}$ has no solutions. Without loss of generality, we shall assume that $p<q$.

Theorem 2.1. Suppose that $2 \leq p<q$ are primes. Then the equation $p^{4}+q^{4}=z^{2}$ has no solutions.

Proof: First we consider the case $p=2$, and then the case $p>2$.

Suppose $p=2$. We have

$$
16+q^{4}=z^{2}, \quad z \text { is odd. }
$$

From (1) it follows that $z^{2}-q^{4}=16$ or

$$
\left(z-q^{2}\right)\left(z+q^{2}\right)=2^{4} .
$$

The five divisors of $2^{4}$ are $1,2^{1}, 2^{2}, 2^{3}, 2^{4}$. Thus from (2) we obtain

$$
z-q^{2}=1 \text { and } z+q^{2}=2^{4}, \quad z-q^{2}=2^{1} \quad \text { and } \quad z+q^{2}=2^{3},
$$

where the last three possibilities are a priori eliminated.

The case $z-q^{2}=1$ and $z+q^{2}=16$ is a priori impossible, since the difference of two odd integers $z$ and $q^{2}$ is never equal to 1 . Hence, this case does not exist.

If $z-q^{2}=2$ and $z+q^{2}=8$, then $z=q^{2}+2$ or $2 q^{2}+2=8$ and $q^{2}=3$ is impossible.

Thus, $p \neq 2$ and $16+q^{4}=z^{2}$ has no solutions.

Suppose $p>2$. The equation $p^{4}+q^{4}=z^{2}$ yields

$$
p^{4}=z^{2}-q^{4}=\left(z-q^{2}\right)\left(z+q^{2}\right) .
$$

The five divisors of $p^{4}$ are $1, p^{1}, p^{2}, p^{3}, p^{4}$. Hence, from (3) we have

$$
z-q^{2}=1 \quad \text { and } z+q^{2}=p^{4}, \quad z-q^{2}=p^{1} \quad \text { and } \quad z+q^{2}=p^{3},
$$

where the last three possibilities are a priori eliminated.

$$
\text { If } z-q^{2}=1 \text { and } z+q^{2}=p^{4} \text {, then } z=q^{2}+1 \text { and } 2 q^{2}+1=p^{4} \text {. Hence, }
$$

The right side of (4) is clearly a multiple of at least 4 , whereas $2 q^{2}$ is a multiple of exactly 2 . This contradiction implies that the first possibility is impossible. 
On Solutions of the Diophantine Equations $p^{4}+q^{4}=z^{2}$ and $p^{4}-q^{4}=z^{2}$ when $p$ and $q$ are Primes

If $z-q^{2}=p$ and $z+q^{2}=p^{3}$, then $z=q^{2}+p$ and $2 q^{2}+p=p^{3}$. Since $\operatorname{gcd}(p, q)=$ 1, it follows that $2 q^{2}+p=p^{3}$ does not exist, and this possibility is also impossible.

Hence, when $p>2, p^{4}+q^{4}=z^{2}$ has no solutions as asserted.

This concludes the proof of Theorem 2.1.

\section{Solutions of the equation $p^{4}-q^{4}=z^{2}$}

In this section we establish: First, for $q=2$ the equation has no solutions. Secondly, for all primes $q>2$ and $p<2100$, the equation also has no solutions.

Theorem 3.1. Suppose that $2 \leq q<p$ are primes. Then the equation $p^{4}-q^{4}=z^{2}$ has no solutions

(i) when $2=q$,

(ii) when $2<q<p<2100$.

Proof: (i) When $q=2$, we have

$p^{4}-16=z^{2}$
From (5) it follows that $p^{4}-z^{2}=16$ or $z$ is odd.

$\left(p^{2}-z\right)\left(p^{2}+z\right)=2^{4}$.

The five divisors of $2^{4}$ are $1,2^{1}, 2^{2}, 2^{3}, 2^{4}$. Thus from (6) we have

$$
p^{2}-z=1 \text { and } p^{2}+z=2^{4}, \quad p^{2}-z=2^{1} \text { and } p^{2}+z=2^{3} \text {, }
$$

where the last three possibilities are a priori eliminated.

The case $p^{2}-z=1$ and $p^{2}+z=16$ is a priori impossible, since the difference of two odd integers $p^{2}$ and $z$ is never equal to 1 . The first possibility does not exist.

If $p^{2}-z=2$ and $p^{2}+z=8$, then $p^{2}=z+2$ or $2 z+2=8$ and $z=3$. But, when $z=3$ then $p^{2}=5$ which is impossible. Hence, the second possibility does not exist.

Thus $q \neq 2$, and $p^{4}-16=z^{2}$ has no solutions. Case (i) is complete.

When $q<p$, then $p^{4}-q^{4}=z^{2}$ yields

$$
p^{4}-q^{4}=\left(p^{2}-q^{2}\right)\left(p^{2}+q^{2}\right)=z^{2} \text {. }
$$

Let $R>1$ be an integer which is not a square. Equation (7) has two possibilities, namely
(a) $p^{2}-q^{2}=A^{2}$
and $\quad p^{2}+q^{2}=B^{2}$,
(b) $p^{2}-q^{2}=C^{2} \cdot R$ and $p^{2}+q^{2}=D^{2} \cdot R$.

(a) Suppose that $p^{2}-q^{2}=A^{2}$ and $p^{2}+q^{2}=B^{2}$.

The values $A^{2}, B^{2}$ are even, hence $A, B$ are even. Denote $A^{2}=4 N$ and $B^{2}=4 M$. Thus, the sum of the two equalities in our supposition yields

$$
2 p^{2}=A^{2}+B^{2}=4 N+4 M=4(N+M)
$$




\section{Nechemia Burshtein}

which is impossible since $p$ is an odd prime.

Case (a) does not exist.

(b) Suppose that $p^{2}-q^{2}=C^{2} R$ and $p^{2}+q^{2}=D^{2} R$.

The sum of the equalities in our supposition yields

$$
2 p^{2}=R\left(C^{2}+D^{2}\right) \text {. }
$$

From (8) it follows that $R \mid 2 p^{2}$, and since $\operatorname{gcd}(R, p)=1$, therefore $R=2$. Hence

from which

$$
p^{2}-q^{2}=2 C^{2} \quad \text { and } \quad p^{2}+q^{2}=2 D^{2},
$$

$$
p^{2}=C^{2}+D^{2} \text { and } q^{2}=D^{2}-C^{2} .
$$

Since $q^{2}=D^{2}-C^{2}=(D-C)(D+C)$ and $q$ is prime, it follows that $D-C=1$ and $D$ $+C=q^{2}$ implying that $q^{2}=2 C+1$. The value $q^{2}-1=(q-1)(q+1)=2 C$ yields for all primes $q$ that $C=4 T$. We have $p^{2}-q^{2}=2 C^{2}$ or $p^{2}=2 C^{2}+q^{2}=2 C^{2}+(2 C+1)=$ $C^{2}+(C+1)^{2}$. Thus,

$$
q^{2}=C+(C+1), \quad p^{2}=C^{2}+(C+1)^{2}, \quad C=4 T .
$$

The equalities in (9) must be satisfied simultaneously with primes $p, q$.

Suppose (ii) $2<q<p<2100$.

In (9), the equality $C^{2}+(C+1)^{2}=p^{2}$ suggests the use of "Pythagorean triples" (abbreviated triples) denoted $(a, b, c)$ if $a^{2}+b^{2}=c^{2}$. All the triples of the form $C^{2}+$ $(C+1)^{2}=p^{2}$ where $p<2100$ have been examined in [5]. There is exactly one triple of this form which satisfies only two of the three conditions in (9). The triple is demonstrated in Table 1. Hence, for all primes $2<q<p<2100$ the three conditions in (9) are not met simultaneously, and $p^{4}-q^{4}=z^{2}$ has no solutions.

This completes part (ii), and Theorem 3.1.

For the convenience of the reader, values $C, q^{2}, p^{2}$ are presented in the following Table 1.

Table 1. Values of $C, q^{2}$ and $p^{2}$

\begin{tabular}{|c|c|c|c|c|}
\hline $\boldsymbol{T}$ & $\boldsymbol{C = 4 T}$ & $\boldsymbol{C + 1 = 4 T + 1}$ & $\boldsymbol{C}+(\boldsymbol{C}+\mathbf{1})=\boldsymbol{q}^{\mathbf{2}}$ & $\boldsymbol{C}^{\mathbf{2}}+(\boldsymbol{C}+\mathbf{1})^{\mathbf{2}}=\boldsymbol{p}^{\mathbf{2}}$ \\
\hline 1 & $C=4$ & $C+1=5$ & $4+5=3^{2}=q^{2}$ & $4^{2}+5^{2}=41 \neq p^{2}$ \\
\hline 3 & $C=12$ & $C+1=13$ & $12+13=5^{2}=q^{2}$ & $12^{2}+13^{2}=313 \neq p^{2}$ \\
\hline 6 & $C=24$ & $C+1=25$ & $24+25=7^{2}=q^{2}$ & $24^{2}+25^{2}=1201 \neq p^{2}$ \\
\hline 15 & $C=60$ & $C+1=61$ & $60+61=11^{2}=q^{2}$ & $60^{2}+61^{2}=7321 \neq p^{2}$ \\
\hline 21 & $C=84$ & $C+1=85$ & $84+85=13^{2}=q^{2}$ & $84^{2}+85^{2}=14281 \neq p^{2}$ \\
\hline & & & & \\
\hline 5 & $C=20$ & $C+1=21$ & $20+21=41 \neq q^{2}$ & $20^{2}+21^{2}=29^{2}=p^{2}$ \\
\hline
\end{tabular}


On Solutions of the Diophantine Equations $p^{4}+q^{4}=z^{2}$ and $p^{4}-q^{4}=z^{2}$ when $p$ and $q$ are Primes

Remark 3.1. It is needless to say, that a priori it seems impossible that the three conditions in (9) may be satisfied simultaneously.

The validity of this statement was verified for all primes $p<2100$.

We can now sum up the results for both equations as follows:

Conjecture 1. For all primes $p>2100$ and $q>2$, the equation $p^{4}-q^{4}=z^{2}$ has no solutions.

If Conjecture $\mathbf{1}$ is indeed true, then both equations $p^{4}+q^{4}=z^{2}$ and $p^{4}-q^{4}=z^{2}$ have no solutions.

\section{REFERENCES}

1. N. Burshtein, On solutions to the diophantine equation $x^{4}-y^{2}=z^{2}$, Annals of Pure and Applied Mathematics, 18 (1) (2018) 79 - 81.

2. N. Burshtein, On solutions of the diophantine equations $p^{3}+q^{3}=z^{2}$ and $p^{3}-q^{3}=z^{2}$ when $p, q$ are primes, Annals of Pure and Applied Mathematics, 18 (1) (2018) $51-57$.

3. N. Burshtein, All the solutions of the diophantine equation $p^{4}+q^{2}=z^{2}$, Annals of Pure and Applied Mathematics, 14 (3) (2017) 457 -459.

4. B. Poonen, Some diophantine equations of the form $x^{n}+y^{n}=z^{m}$, Acta Arith., 86 (1998) 193-205.

5. Integer Lists: "Pythagorean triples up to 2100" - TSM Resources. 